\title{
醋酸碘苯介导的 $\alpha$-重氮羰基化合物的去重氮双氧合反应
}

\author{
李庆雪 李梦伟 ${ }^{\dagger}$ 时绍青 ${ }^{\dagger}$ 季晓霜 ${ }^{\dagger}$ 何春兰 \\ 姜 波* 郝文娟* \\ (江苏师范大学化学与材料科学学院 徐州 221116)
}

\begin{abstract}
摘要 报道了一类新颖的醋酸碘苯介导的 $\alpha$-重氮羰基化合物的去重氮双氧合反应. 该反应利用醋酸碘苯与 $N$-羟基邻苯 二甲酰亚胺(或 $N$-羊基丁二甲酰亚胺)反应能产生氧中心自由基的特性实现了氧中心自由基诱导的 $\alpha$-重氮羰基化合物的 双氧合反应, 合成了一系列 $\alpha, \alpha$-双氧代芳酮和 $\alpha, \alpha$-双氧代羧酸酯衍生物, 产率中等到良好. 基于实验结果及文献报道, 提出了可能的反应机理，其涉及氧中心自由基加成、 $\mathrm{C}-\mathrm{N}$ 键的均裂和自由基交叉偶联等. 此外, 该反应具有无需金属 催化剂、条件温和、操作简便等优点.
\end{abstract}

关键词 双氧合反应; 去重氮化反应; $\alpha, \alpha$-双氧代芳酮; $\alpha, \alpha$-双氧代羧酸酯

\section{$\mathrm{Phl}(\mathrm{OAc})_{2}$-Mediated Dediazodioxygenation of $\alpha$-Diazo Carbonyls}

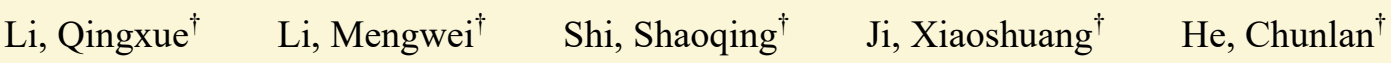 \\ Jiang, Bo* Hao, Wenjuan* \\ (School of Chemistry \& Materials Science, Jiangsu Normal University, Xuzhou 221116)
}

\begin{abstract}
A new $\mathrm{PhI}(\mathrm{OAc})_{2}$-mediated dediazodioxygenation of $\alpha$-diazo carbonyls was reported. By using the characteristics of the in-situ-generated $\mathrm{O}$-centered radicals from the interaction of $\mathrm{PhI}(\mathrm{OAc})_{2}$ and $\mathrm{N}$-hydroxy phthalimide (or $\mathrm{N}$-hydroxy succinimide), $O$-centered radical-triggered dioxygenation of $\alpha$-diazo carbonyls was achieved in this transformation, which led to the synthesis of a series of $\alpha, \alpha$-dioxoarylketones and $\alpha, \alpha$-dioxoesters with moderate to good yields. Based on the experimental results and literature reports, the possible reaction mechanism was proposed, which involved $O$-centered radical addition, $\mathrm{C}-\mathrm{N}$ bond homolysis and radical cross coupling. In addition, the reaction featured mild conditions and simple operation without any catalyst.
\end{abstract}

Keywords dioxygenation; dediazotization; $\alpha, \alpha$-dioxoarylketones; $\alpha, \alpha$-dioxoesters

重氮化合物具有高反应活性, 是一种多用途的有机 砌块, 广泛应用于有机合成、化学生物学、材料化学、 药物化学等领域 ${ }^{[1]}$. 在重氮单元的 $\alpha$-位引入强吸电子基 团如羰基，可分散与重氮基相连碳上的负电荷，增加了 重氮单元的稳定性, 该结构特征能使 $\alpha$-重氮羰基化合物 在常温下稳定存放. 由于其易于制备且展示出优秀的化 学性质, $\alpha$-重氮羰基化合物在合成官能化羰基化合物中 往往是首选底物, 在有机合成中主要表现为: 在光或热 条件下使其成为自由卡宾(free carbene), 在过渡金属催 化下能形成金属卡宾(metal carbene). 这两种卡宾中间 体都具有非常高的反应活性, 可发生各种各样的经典转
化, 如 $\mathrm{X}-\mathrm{H}$ 插入反应 ${ }^{[2 \sim 4]}(\mathrm{X}=\mathrm{C} 、 \mathrm{O} 、 \mathrm{~N}$ 等 $)$ 、环丙烷化 反应 ${ }^{[5]}$ 、叶立德反应 ${ }^{[6]}$ 、wolff 重排反应 ${ }^{[7]}$ 以及多组分反 应 $^{[8]}$.

自由基转化是一类非常重要的反应类型，通过自由 基的引发串联, 将较简单的原料经过很短的步骤连续发 生多个化学键的均裂和形成, 转化成复杂的分子, 它具 有反应过程简捷, 无需分离中间体, 成键高效等优点 ${ }^{[9]}$. 自由基反应已成为一种能在较为温和条件下合成某些 特定复杂有机分子的强有力工具 ${ }^{[10]}$. 最近研究表明重 氮化合物可作为自由基受体, 能与各种自由基物种发生 加成反应，构建一些具有重要生物活性的分子骨架 ${ }^{[11]}$.

* Corresponding authors. E-mail: jiangchem@jsnu.edu.cn; wjhao@jsnu.edu.cn

Received September 30, 2019; revised October 15, 2019; published online November 1, 2019.

Project supported by the National Natural Science Foundation of China (No. 21602087), the Top-Notch Academic Programs Project of Jiangsu Higher Education Institutions and the National College Student's Innovation and Entrepreneurship Training Program (Nos. 201810320156X, 201810320019Z).

国家自然科学基金(No. 21602087)、江苏省品牌专业基金和国家级大学生创新创业训练计划(Nos. 201810320156X, 201810320019Z)资助项目.

$\dagger$ 共同第一作者(These authors contributed equally to this work). 
苏州大学万小兵课题组 ${ }^{[12]}$ 用叔丁基过氧化氢(TBHP)作 为引发剂将叔胺氧化, 使其形成 $\alpha$-氨烷基自由基物种, 在钴催化下与重氮化合物和苯乙烯发生多组分氧化偶 联反应, 合成了一系列新型的 $\gamma$-羰基- $\beta$-氨酯衍生物. 该 反应过程涉及金属钴与重氮化合物原位形成钴卡宾自 由基物种, 再与 $\alpha$-氨烷基自由基发生自由基交叉偶联 (Scheme 1a). 最近, 我们课题组 ${ }^{[13]}$ 使用 $\alpha$-重氮芳酮、 $N$ 羟基邻苯二甲酰亚胺和 $N, N-$ 二甲基苯胺为原料, 在醋酸 碘苯介导下进行自由基三组分反应, 合成了一系列 $\alpha$-氨 氧基- $\beta$-氨基酮衍生物, 实现了无金属催化的 $\alpha$-重氮芳 酮的去重氮碳氧合反应. 继续我们在 $\alpha$-重氮羰基化合物 的去重氮双官能化研究, 我们认为, 在适当氧化条件下 $N$-差基邻苯二甲酰亚胺(NHPI)原位转化为 $N$-氧邻苯二 甲酰亚胺自由基(PINO), 若用 $\alpha$-重氮羰基化合物进行捕 捉, 随后脱氮生成 $\alpha$-羰烷基自由基, 再与另一组分 PINO 发生自由基偶联, 合成得到新型的双异吲哚啉二 酮衍生物, 实现了 $\alpha$-重氮羰基化合物的去重氮双氧合化 反应. 与预期一致, 该反应在醋酸碘苯介导下可有效进 行, 得到相应的双异吲哚啉二酮衍生物 3 (Scheme 1b). 用 $N$-羟基丁二酰亚胺作为氧自由基供体时, 此反应在 醋酸碘苯介导下也能平稳进行, 得到相应的双丁二酰亚 胺衍生物. 此外, $\alpha$-芳基重氮酯也可参与此反应, 得到 一系列含有季碳中心的双异吲哚啉二酮衍生物.

(a) Wan's work

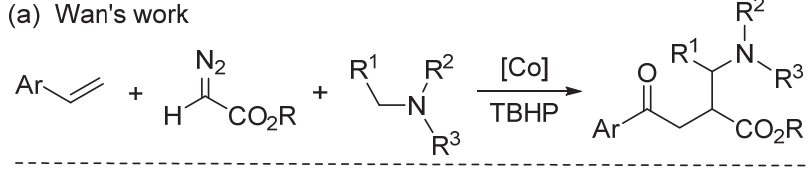

(b) this work<smiles></smiles><smiles>[R]C(=O)C([R])(ON1C(=O)c2ccccc2C1=O)ON1C(=O)c2ccccc2C1=O</smiles>

图式 $1 \alpha$-重氮羰基化合物的自由基加成反应 Scheme 1 Radical addition of $\alpha$-diazo carbonyls

\section{1 结果与讨论}

\section{1 反应条件的优化}

以对甲苯甲酰重氮(1a)、 $N$-羟基邻苯二甲酰亚胺 (NHPI, 2a) 为模板底物, 研究投料比、不同氧化剂及其用
量、不同溶剂和不同温度对产物产率的影响. 实验结果 见表 1. 首先对底物投料比进行了篮选(表 1, Entries 1 3). 当底物 $1 \mathrm{a}$ 与 $2 \mathrm{a}$ 的物料比为 $1: 2$ 时, 该反应以 4.0 equiv. 的醋酸碘苯(PIDA) 为氧化剂和 1,2-二氯乙烷(DCE) 为溶剂在室温下能顺利进行, 以 $34 \%$ 收率地给出双异吲 哚啉二酮产物 $3 \mathbf{a}$ (表 1, Entry 1). 由于在上述反应中发现 底物 $2 \mathrm{a}$ 有剩余, 因此增加 $1 \mathrm{a}$ 的用量, 将其投料比调整 为 $1.2: 2$ 和 $1.5: 2$ 进行尝试, 发现收率有明显上升(表 1, Entries 2 3). 其中投料比为 $1.5: 2$ 时, 产物 3a 的收 率上升到 58\%(表 1, Entry 3). 接下来考察反应溶剂对反 应的影响. 当使用乙腈为溶剂时, 产率大幅下降, 仅有 $23 \%$ (表 1, Entry 4), 而使用甲醇和甲苯作溶剂时，仅有 少量目标产物被检测到(表 1, Entries 5 6). 随后选择 DCE 为溶剂对醋酸碘苯的用量进行了篮选, 发现只有 用量为 4.0 equiv. 时, 反应效果最优, 增加或者减少醋酸 碘苯的用量都会导致产率降低(表 1, Entries 7～8). 考察 反应温度发现最佳反应温度仍为室温(表 1, Entries 9 10). 最后采用双(三氟乙酰氧基)碘苯(PIFA)作为氧化剂, 不能得到相应的目标产物(表 1, Entry 11). 综上所述, 最 优条件是 $1 \mathrm{a}$ 与 $2 \mathrm{a}$ 的投料比为 $1.5: 2,4.0$ equiv. 的醋酸 碘苯为氧化剂, DCE 为溶剂, 以及室温为反应温度.

表 1 化合物 3a 反应条件的优化 ${ }^{a}$

Table 1 Optimization of reaction conditions for the synthesis of $3 a$
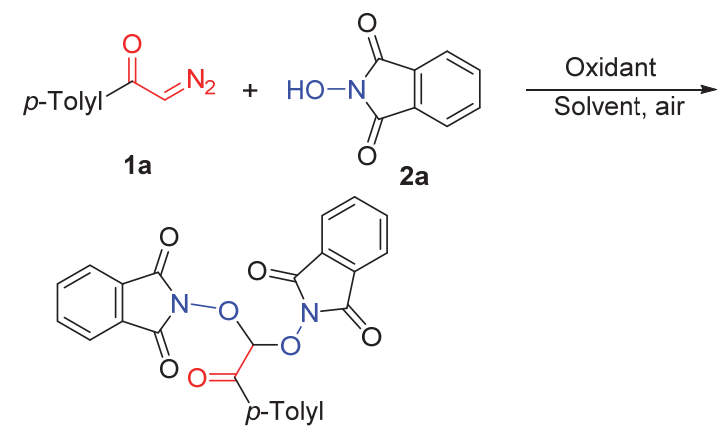

3a

\begin{tabular}{ccccc}
\hline Entry & Oxidant (equiv.) & Solvent & $T /^{\circ} \mathrm{C}$ & Yield $^{b} \%$ \\
\hline 1 & PIDA (4.0) & DCE & r.t. & $34^{c}$ \\
2 & PIDA (4.0) & DCE & r.t. & $50^{d}$ \\
3 & PIDA (4.0) & DCE & r.t. & $58^{e}$ \\
4 & PIDA (4.0) & MeCN & r.t. & 23 \\
5 & PIDA (4.0) & MeOH & r.t. & Trace \\
6 & PIDA (4.0) & Toluene & r.t. & Trace \\
7 & PIDA (3.0) & DCE & r.t. & 12 \\
8 & PIDA (6.0) & DCE & r.t. & 24 \\
9 & PIDA (4.0) & DCE & 40 & 35 \\
10 & PIDA (4.0) & DCE & 60 & 32 \\
11 & PIFA (4.0) & DCE & r.t. & Trace \\
\hline
\end{tabular}

${ }^{a}$ Reaction conditions: $\alpha$-diazoketones (1a, $\left.0.25 \mathrm{mmol}\right)$, NHPI (2, $0.5 \mathrm{mmol}$ ), oxidant, solvent $(2.0 \mathrm{~mL})$, air. ${ }^{b}$ Isolated yield based on NHPI. ${ }^{c}$ The ratio of $\mathbf{1 a}: \mathbf{2}$ is $1: 2 .{ }^{d}$ The ratio of $\mathbf{1 a}: \mathbf{2}$ is $1.2: 2 .{ }^{e}$ The ratio of $\mathbf{1 a}: \mathbf{2}$ is $1.5: 2$. 


\section{2 反应底物的拓展}

在最佳优化条件下，对该方法普适性进行了探索 (表 2). 最初以 $N$-羟基邻苯二甲酰亚胺(2a)为代表性底 物考察了 $\alpha$-重氮羰基化合物上取代基 $\mathrm{R}^{1}$ 与 $\mathrm{R}^{2}$ 的适用范 围. 结果表明: $R^{1}$ 为芳环及 $R^{2}$ 为 $H$ 时, 多种与苯环相连 的取代基均能兼容此反应，如供电子基团的甲氧基(1b) 和吸电子基团的氯(1d). 从实验结果可以得出, 芳基上 基团的电子效应对产物收率影响不明显. 此外, 无取代 基的苯基(1c)也能以中等收率得到目标产物 3c. 值得一 提的是, 重氮乙酸乙酯 $(1 \mathrm{e})$ 在此反应中被证实是一类良 好的自由基受体，提供相应的目标产物 3e, 收率为 51\%。 随后, 用 $N$-差弪基丁二酰亚胺 $(\mathbf{2} \mathbf{b})$ 代替 $N$-着基邻苯二甲酰 亚胺来评估反应的适用范围. 固定 $\mathrm{R}^{2}$ 为氢且调整 $\alpha$-重 氮羰基化合物上的取代基 $\mathrm{R}^{1}$ 为对甲苯基(1a)、对甲氧苯 基(1b)、苯基(1c)、对氯苯基(1d)及对苯溴基(1f)时发现, 上述取代基均可参与此反应, 得到目标产物 $\mathbf{3 f} \sim \mathbf{3 k}$, 产

表 2 化合物 3a $\sim 3 \mathrm{t}$ 的合成

Table 2 Synthesis of products $\mathbf{3 a} \sim \mathbf{3}$

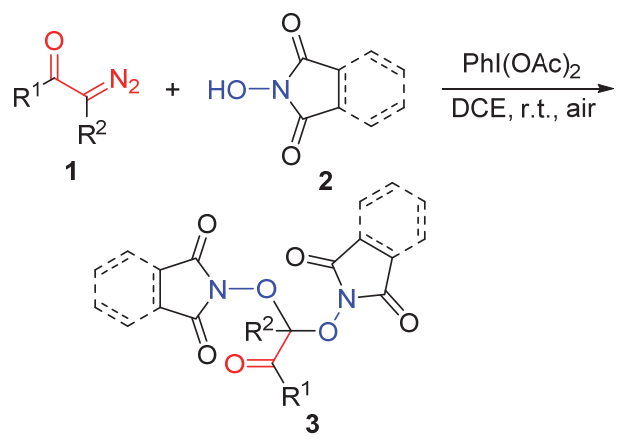

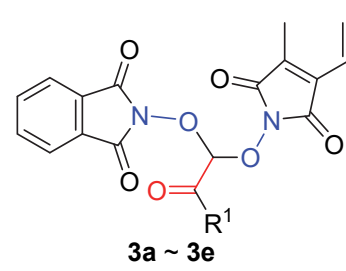

3a, $\mathrm{R}^{1}=p$-Tolyl $(58 \%)$

3b, $\mathrm{R}^{1}=4-\mathrm{MeOC}_{6} \mathrm{H}_{4}(52 \%)$

3c, $\mathrm{R}^{1}=\mathrm{Ph}(53 \%)$

3d, $\mathrm{R}^{1}=4-\mathrm{ClC}_{6} \mathrm{H}_{4}(56 \%)$

3e, $\mathrm{R}^{1}=\mathrm{OEt}(51 \%)$

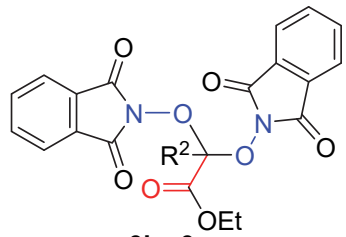

$$
\text { 3) } \sim 3 \mathbf{p}
$$

3I, $\mathrm{R}^{2}=4-\mathrm{MeOC}_{6} \mathrm{H}_{4}(56 \%)$

$3 \mathrm{~m}, \mathrm{R}^{2}=\mathrm{Ph}(46 \%)$

3n, $\mathrm{R}^{2}=4-\mathrm{FC}_{6} \mathrm{H}_{4}(57 \%)$

3o, $\mathrm{R}^{2}=4-\mathrm{ClC}_{6} \mathrm{H}_{4}(49 \%)$

$3 p, \mathrm{R}^{2}=4-\mathrm{BrC}_{6} \mathrm{H}_{4}(53 \%)$
率在 $50 \% \sim 87 \%$ 之间. 其中 $\alpha$-重氮芳酮 $1 \mathrm{a}$ 与重氮乙酸乙 酯(1e)给出目标产物 $3 \mathrm{f}$ 和 $3 \mathrm{k}$ 的收率相对较低, 其它官能 才给出的收率较高，在 79\% 87\%之间 $(\mathbf{3 g} \sim \mathbf{3 j})$. 为了继 续拓展反应的适用范围，使用多种取代不同的 $\alpha$-芳基重 氮酯 $1 \mathrm{~g} \sim 1 \mathrm{k}$, 如对甲氧苯基 $(1 \mathrm{~g})$ 、苯基 $(1 \mathrm{~h})$ 、对氟苯基 (1i)、对氯苯基 $(1 \mathrm{j})$ 及对苯溴基(1k), 分别与 $2 \mathrm{a}$ 和 $2 \mathrm{~b}$ 进 行反应. 实验结果表明, 上述底物 $1 \mathrm{~g} \sim 1 \mathrm{k}$ 均能顺利参与 此反应，得到一系列含有季碳中心的双氧合产物 $31 \sim 3 t$, 收率为 $46 \% \sim 60 \%$. 收率低的原因可能是由于其空间位 阻造成的. 所合成的产物 3 的结构均经 NMR 谱和 HRMS 表征. 此外, 通过单晶 X 射线衍射进一步确定了 化合物 3a 的结构(图 1), 英国剑桥晶体数据库(CCDC) 号为 1956604 .

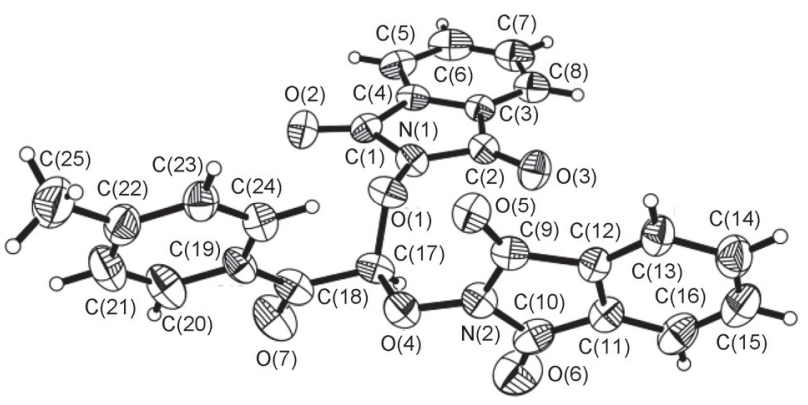

图 1 双异吲哚啉二酮衍生物 3a 的晶体结构图

Figure 1 X-ray structure of bis-isoindoline-1,3-dione 3a

\section{3 反应机理探讨}

为了探究反应机理，向两个独立的反应体系中分别 加入自由基捕获剂四甲基哌啶氧化物(TEMPO)和抑制 剂 2,6-二叔丁基-4-甲基苯酚(BHT), 我们发现反应体系 被抑制，没有检测到目标产物 3a (Scheme 2). 由此可推 断该反应过程中可能涉及自由基历程.

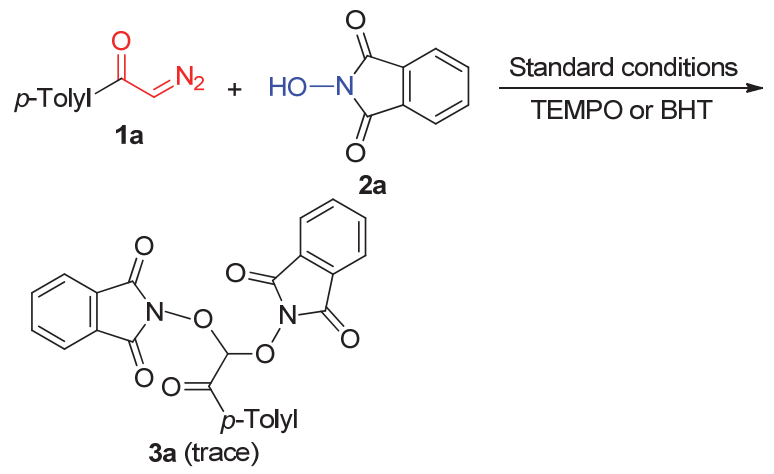

图式 2 控制反应

Scheme 2 Control experiment

根据已有的文献报道 ${ }^{[12,13]}$ 与上述的控制反应，提出 $3 q, R^{2}=P h(54 \%)$ $3 \mathbf{r}, \mathrm{R}^{2}=4-\mathrm{FC}_{6} \mathrm{H}_{4}(60 \%)$ 3s, $\mathrm{R}^{2}=4-\mathrm{ClC}_{6} \mathrm{H}_{4}(56 \%)$ 3t, $\mathrm{R}^{2}=4-\mathrm{BrC}_{6} \mathrm{H}_{4}(52 \%)$ 
基物种 $\mathbf{B}$ 再算取 NHPI 的羟基上的 H 也可形成 PINO 自 由基. 随后, PINO 自由基与重氮化合物进行加成, 形成 重氮自由基 $\mathbf{C}$, 脱去一分子氮气产生 $\alpha$-羰烷基自由基 $\mathbf{D}$. 最后, $\mathbf{D}$ 与 PINO 进行自由基偶联得到最终的目标产物.

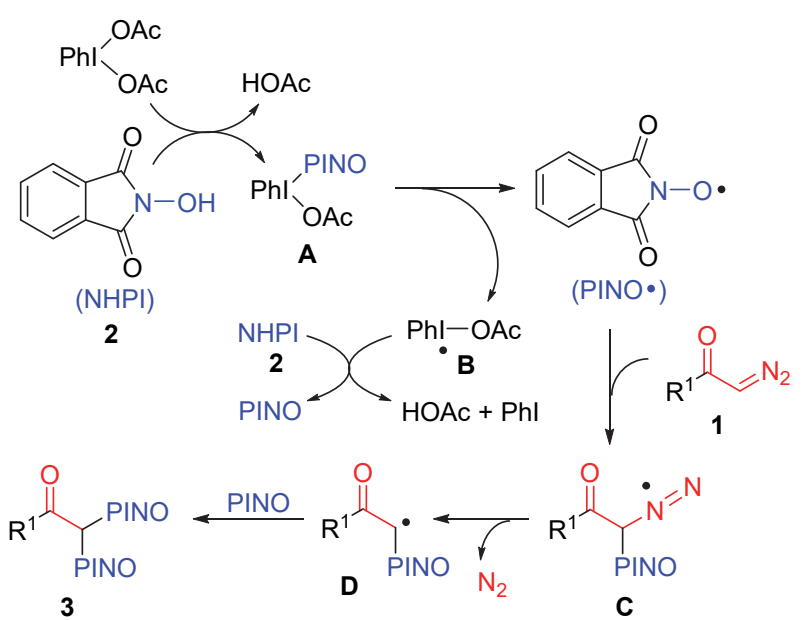

图式 3 生成化合物 $\mathbf{3}$ 的可能机理

Scheme 3 Proposed mechanism for the synthesis of products 3

\section{2 结论}

在醋酸碘苯介导条件下, 将 1 分子的 $a$-重氮羰基化 合物与 2 分子的 $N$-着基邻苯二甲酰亚胺(或 $N$-羟基丁二 甲酰亚胺)进行多组分反应, 经历自由基加成、脱重氮及 自由基交叉偶联过程, 合成了 20 个 $\alpha, \alpha$-双氧代芳酮或 $\alpha, \alpha$-双氧代酸酯衍生物，产率为 $46 \% \sim 87 \%$. 该反应实 现了温和条件下的碳氮双键的切割和两个碳氧单键的 构建, 完成了双异吲哚啉-1,3-二酮和双丁二甲酰亚胺骨 架有效构筑, 为去重氮双官能化反应提供了一种有效的 策略方法

\section{3 实验部分}

\section{1 仪器与试剂}

仪器: 熔点测定仪 (XT-5 型); 红外光谱仪 (FTIRTensor 27 型); 质谱仪(Bruker microTOF-Q II 型); 核磁 共振仪(Bruker DPX $400 \mathrm{MHz}$ 型); 上海暗箱紫外分析仪 (ZF-20D 型); 四圆衍射仪(Siemens P4 型)等.

试剂: DMSO- $d_{6}$ ( 内标为 $\mathrm{TMS}$ )、 $\mathrm{CDCl}_{3}$ (内标为 TMS)、乙酸乙酯(分析纯)、石油梄(分析纯)、乙腈(分析 纯)、 $N$-差基丁二酰亚胺、 $N$-羟基邻苯酰亚胺等.

\section{2 实验方法}

向 10-mL Schlenk 反应管中加入 $N$-羟基邻苯二甲酰 亚胺 $(1 \mathrm{mmol}$ ), 醋酸碘苯 (4 equiv.), 再加 $1.5 \mathrm{~mL}$ DCE. 随后将 $\alpha$-重氮羰基化合物 $(0.75 \mathrm{mmol})$ 加入到体系中, 室 温下进行反应, $12 \mathrm{~h}$ 后, 用薄层色谱(TLC)跟踪检测. 反
应结束后, 将体系转移至 $50 \mathrm{~mL}$ 圆底烧瓶中减压蒸馏, 通过柱层析 $[V$ (石油醚) $: V$ (乙酸乙酯 $)=1: 15]$, 分离得 到化合物 3a $\sim 3 \mathbf{t}$.

2,2'-((2-氧代-2-(4-甲苯基)乙烷-1,1-二基)双(氧基)) 双 (二氢异吲哚啉-1,3-二酮)(3a): $132 \mathrm{mg}$ 白色固体, 产率 58\%. m.p. $215 \sim 216{ }^{\circ} \mathrm{C} ;{ }^{1} \mathrm{H}$ NMR (400 MHz, $\left.\mathrm{CDCl}_{3}\right) \delta$ : $8.42(\mathrm{~d}, J=8.4 \mathrm{~Hz}, 2 \mathrm{H}), 7.87 \sim 7.78(\mathrm{~m}, 8 \mathrm{H}), 7.42(\mathrm{~d}, J=$ $8.4 \mathrm{~Hz}, 2 \mathrm{H}), 6.09$ (s, 1H), 2.49 (s, 3H) ${ }^{13} \mathrm{C}$ NMR $(100$ $\left.\mathrm{MHz}, \mathrm{CDCl}_{3}\right) \delta: 187.1,162.4,145.8,134.8,130.6,130.0$, 129.6, 128.7, 123.9, 112.3, 22.0; IR (KBr) v: 3123, 1733, 1697, 1399, 1280, 1188, 982, 876, $696 \mathrm{~cm}^{-1}$; HRMS (ESI-TOF) calcd for $\mathrm{C}_{25} \mathrm{H}_{16} \mathrm{~N}_{2} \mathrm{NaO}_{7}[\mathrm{M}+\mathrm{Na}]^{+}$479.0855, found 479.0866 .

2,2'-((2-氧代-2-(4-甲氧苯基)乙烷-1,1-二基)双(氧 基))双(二氢异吲哚啉-1,3-二酮)(3b): $123 \mathrm{mg}$ 白色固体, 产率 52\%. m.p. $193 \sim 194{ }^{\circ} \mathrm{C}$; ${ }^{1} \mathrm{H}$ NMR $(400 \mathrm{MHz}$, $\left.\mathrm{CDCl}_{3}\right) \delta: 8.52(\mathrm{~d}, J=8.8 \mathrm{~Hz}, 2 \mathrm{H}), 7.84 \sim 7.78(\mathrm{~m}, 8 \mathrm{H})$, 7.09 (d, $J=8.8 \mathrm{~Hz}, 2 \mathrm{H}), 6.07$ (s, 1H), 3.94 (s, 3H); ${ }^{13} \mathrm{C}$ NMR (100 MHz, $\left.\mathrm{CDCl}_{3}\right) \delta: 185.9,164.7,162.4,134.8$, 133.1, 128.7, 125.4, 123.9, 114.1, 112.6, 55.6; IR (KBr) v: 3121, 1732, 1685, 1506, 1400, 1206, 1062, 999, 833, 668 $\mathrm{cm}^{-1}$; HRMS (ESI-TOF) calcd for $\mathrm{C}_{25} \mathrm{H}_{16} \mathrm{~N}_{2} \mathrm{NaO}_{8}[\mathrm{M}+$ $\mathrm{Na}]^{+}$495.0804, found 495.0806.

2,2'-((2-氧代-2-苯基乙烷-1,1-二基)双(氧基))双 (二 氢异吲哚啉-1,3-二酮)(3c): $117 \mathrm{mg}$, 白色固体, 产率 53\%. m.p. 197 $198{ }^{\circ} \mathrm{C} ;{ }^{1} \mathrm{H}$ NMR (400 MHz, $\left.\mathrm{CDCl}_{3}\right) \delta$ : $8.52(\mathrm{~d}, J=7.6 \mathrm{~Hz}, 2 \mathrm{H}), 7.80 \sim 7.78(\mathrm{~m}, 8 \mathrm{H}), 7.73 \sim 7.58$ $(\mathrm{m}, 3 \mathrm{H}), 6.11(\mathrm{~s}, 1 \mathrm{H}) ;{ }^{13} \mathrm{C}$ NMR $\left(100 \mathrm{MHz}, \mathrm{CDCl}_{3}\right) \delta$ : $187.5,162.4,134.8,134.6,132.4,130.5,128.8,128.7$, 123.9, 112.3; IR (KBr) v: 3122, 1792, 1739, 1685, 1446, 1399, 1279, 1188, 1127, 975, $696 \mathrm{~cm}^{-1}$; HRMS (ESI-TOF) calcd for $\mathrm{C}_{24} \mathrm{H}_{14} \mathrm{~N}_{2} \mathrm{NaO}_{7}[\mathrm{M}+\mathrm{Na}]^{+}$465.0699; found 465.0678 .

2,2'-((2-氧代-2-(4-氯苯基)乙烷-1,1-二基)双(氧基)) 双(二氢异吲哚啉-1,3-二酮)(3d): $133 \mathrm{mg}$ 白色固体, 产 率 56\%. m.p. 195 196 ${ }^{\circ} \mathrm{C} ;{ }^{1} \mathrm{H}$ NMR $\left(400 \mathrm{MHz}, \mathrm{CDCl}_{3}\right)$ $\delta: 8.48(\mathrm{~d}, J=8.4 \mathrm{~Hz}, 2 \mathrm{H}), 7.87 \sim 7.85(\mathrm{~m}, 8 \mathrm{H}), 7.60(\mathrm{~d}$, $J=8.4 \mathrm{~Hz}, 2 \mathrm{H}), 6.05$ (s, $1 \mathrm{H}) ;{ }^{13} \mathrm{C}$ NMR $\left(100 \mathrm{MHz}, \mathrm{CDCl}_{3}\right)$ $\delta: 187.5,162.4,134.8,134.6,132.4,130.5,128.8,128.7$, 123.9, 112.3; IR (KBr) v: 3106, 1781, 1739, 1585, 1467, 1355, 1246, 1184, 1055, 999, 875, 787, $696 \mathrm{~cm}^{-1}$; HRMS (ESI-TOF) calcd for $\mathrm{C}_{24} \mathrm{H}_{13} \mathrm{ClN}_{2} \mathrm{NaO}_{7}[\mathrm{M}+\mathrm{Na}]^{+}$ 499.0309, found 499.0302.

2,2-双(2-氧代-(1,3-二氧代异吲哚啉)) 乙酸乙酯(3e): $105 \mathrm{mg}$ 白色固体, 产率 51\%. m.p. $168 \sim 169{ }^{\circ} \mathrm{C} ;{ }^{1} \mathrm{H}$ 
NMR (400 MHz, $\left.\mathrm{CDCl}_{3}\right) \delta: 7.79 \sim 7.77(\mathrm{~m}, 4 \mathrm{H}), 7.71 \sim$ $7.69(\mathrm{~m}, 4 \mathrm{H}), 5.76(\mathrm{~s}, 1 \mathrm{H}), 4.40(\mathrm{~d}, J=7.2 \mathrm{~Hz}, 2 \mathrm{H}), 1.36(\mathrm{t}$, $J=7.2 \mathrm{~Hz}, 3 \mathrm{H}) ;{ }^{13} \mathrm{C} \mathrm{NMR}\left(100 \mathrm{MHz}, \mathrm{CDCl}_{3}\right) \delta: 162.4$, $162.2,134.8,128.7,123.9,105.9,63.2,13.9$; IR (KBr) $v$ : 3117, 2976, 1794, 1732, 1465, 1373, 1213, 1081, 995, 873, $696 \mathrm{~cm}^{-1}$; HRMS (ESI-TOF) calcd for $\mathrm{C}_{20} \mathrm{H}_{14} \mathrm{~N}_{2} \mathrm{NaO}_{8}$ $[\mathrm{M}+\mathrm{Na}]^{+}$433.0648, found 433.0646 .

1,1'-((2-氧代-2-(4-甲苯基)乙烷-1,1-二基)双(氧基)) 双(1H-吡咯-2,5-二酮)(3f)：101 mg 白色固体，产率 56\%. m.p. $207 \sim 208{ }^{\circ} \mathrm{C} ;{ }^{1} \mathrm{H}$ NMR (400 MHz, $\left.\mathrm{CDCl}_{3}\right) \delta$ : 8.31 (d, $J=8.2 \mathrm{~Hz}, 2 \mathrm{H}), 7.36$ (d, $J=8.2 \mathrm{~Hz}, 2 \mathrm{H}), 5.84(\mathrm{~s}, 1 \mathrm{H})$, $2.79(\mathrm{~s}, 8 \mathrm{H}), 2.46(\mathrm{~s}, 3 \mathrm{H}) ;{ }^{13} \mathrm{C} \mathrm{NMR}\left(100 \mathrm{MHz}, \mathrm{CDCl}_{3}\right) \delta$ : 186.6, 170.2, 145.7, 130.5, 129.6, 129.5, 110.9, 25.5, 21.9; IR (KBr) v: 3122, 1792, 1735, 1653, 1540, 1399, 1286, 1206, 1070, 814, $668 \mathrm{~cm}^{-1}$; HRMS (ESI-TOF) calcd for $\mathrm{C}_{17} \mathrm{H}_{16} \mathrm{~N}_{2} \mathrm{NaO}_{7}[\mathrm{M}+\mathrm{Na}]^{+}$383.0855, found 383.0856.

1,1'-((2-氧代-2-(4-甲氧苯基)乙烷-1,1-二基)双(氧 基))双(吡咯烷-2,5-二酤)(3g)：149 mg 白色固体, 产率 79\%. m.p. 205 206 ${ }^{\circ} \mathrm{C}$; ${ }^{1} \mathrm{H}$ NMR (400 MHz, $\left.\mathrm{CDCl}_{3}\right) \delta$ : 8.41 (d, $J=8.8 \mathrm{~Hz}, 2 \mathrm{H}), 7.04$ (d, $J=9.2 \mathrm{~Hz}, 2 \mathrm{H}), 5.81$ (s, $1 \mathrm{H}), 3.92(\mathrm{~s}, 3 \mathrm{H}), 2.80(\mathrm{~s}, 8 \mathrm{H}) ;{ }^{13} \mathrm{C} \mathrm{NMR}(100 \mathrm{MHz}$, $\left.\mathrm{CDCl}_{3}\right) \delta: 170.2,164.7,133.0,114.1,111.2,55.6,25.5$; IR (KBr) $v: 3119,1697,1587,1400,1285,1213,1072,957$, $686 \mathrm{~cm}^{-1}$; HRMS (ESI-TOF) calcd for $\mathrm{C}_{17} \mathrm{H}_{16} \mathrm{~N}_{2} \mathrm{NaO}_{8}$ $[\mathrm{M}+\mathrm{Na}]^{+}$399.0804, found 399.0806.

1,1'-((2-氧代-2-苯基)乙烷-1,1-二基)双(氧基))双(吡 咯烷-2,5-二酮)(3h): $142 \mathrm{mg}$ 白色固体, 产率 82\%. m.p. $218 \sim 219{ }^{\circ} \mathrm{C} ;{ }^{1} \mathrm{H}$ NMR (400 MHz, $\left.\mathrm{CDCl}_{3}\right) \delta: 8.29$ (d, $J=$ $7.6 \mathrm{~Hz}, 2 \mathrm{H}), 7.76$ (d, $J=7.6 \mathrm{~Hz}, 1 \mathrm{H}), 7.63$ (t, $J=8.0 \mathrm{~Hz}$, $2 \mathrm{H}), 6.25(\mathrm{~s}, 1 \mathrm{H}), 2.64(\mathrm{~s}, 8 \mathrm{H}) ;{ }^{13} \mathrm{C}$ NMR $(100 \mathrm{MHz}$, DMSO) $\delta: 187.6,171.6,135.2,133.0,130.1,129.4,108.2$, 26.0; IR (KBr) v: 3122, 1791, 1738, 1699, 1449, 1400, 1191, 1072, 949, 883, 812, $690 \mathrm{~cm}^{-1}$; HRMS (ESI-TOF) calcd for $\mathrm{C}_{16} \mathrm{H}_{14} \mathrm{~N}_{2} \mathrm{NaO}_{7}[\mathrm{M}+\mathrm{Na}]^{+}$369.0699, found 369.0688 .

1,1'-((2-氧代-2-(4-氯苯基)乙烷-1,1-二基)双(氧基)) 双(吡咯烷-2,5-二酮)(3i)：165 mg 白色固体，产率 87\%. m.p. $228 \sim 229{ }^{\circ} \mathrm{C} ;{ }^{1} \mathrm{H}$ NMR (400 $\left.\mathrm{MHz}, \mathrm{CDCl}_{3}\right) \delta: 8.27$ (d, $J=8.8 \mathrm{~Hz}, 2 \mathrm{H}), 7.44$ (d, $J=8.8 \mathrm{~Hz}, 2 \mathrm{H}), 5.70(\mathrm{~s}, 1 \mathrm{H})$, $2.70(\mathrm{~s}, 8 \mathrm{H}) ;{ }^{13} \mathrm{C}$ NMR $\left(100 \mathrm{MHz}, \mathrm{CDCl}_{3}\right) \delta: 186.1,170.1$, $141.2,131.9,130.3,129.2,110.8,25.5$; IR (KBr) v: 3119, 1697, 1587, 1400, 1285, 1213, 1072, 957, $686 \mathrm{~cm}^{-1}$; HRMS (ESI-TOF) calcd for $\mathrm{C}_{16} \mathrm{H}_{13} \mathrm{ClN}_{2} \mathrm{NaO}_{7}[\mathrm{M}+\mathrm{Na}]^{+}$ 403.0309, found 403.0299.

1,1'-((2-氧代-2-(4-溴苯基)乙烷-1,1-二基)双(氧基)) 双(吡咯烷-2,5-二酮)(3j): $178 \mathrm{mg}$ 白色固体，产率 84\%. m.p. $225 \sim 226{ }^{\circ} \mathrm{C} ;{ }^{1} \mathrm{H}$ NMR (400 $\left.\mathrm{MHz}, \mathrm{CDCl}_{3}\right) \delta: 8.20$ $(\mathrm{d}, J=8.8 \mathrm{~Hz}, 2 \mathrm{H}), 7.62(\mathrm{~d}, J=8.8 \mathrm{~Hz}, 2 \mathrm{H}), 5.81(\mathrm{~s}, 1 \mathrm{H})$, $2.80(\mathrm{~s}, 8 \mathrm{H}) ;{ }^{13} \mathrm{C}$ NMR $\left(100 \mathrm{MHz}, \mathrm{CDCl}_{3}\right) \delta: 186.3,170.1$, 132.2, 131.9, 130.8, 130.2, 110.8, 25.5; IR (KBr) v: 3121, 1784, 1724, 1695, 1586, 1430,1196, 1086, 961, $646 \mathrm{~cm}^{-1}$; HRMS (ESI-TOF) calcd for $\mathrm{C}_{16} \mathrm{H}_{13} \mathrm{BrN}_{2} \mathrm{NaO}_{7}[\mathrm{M}+\mathrm{Na}]^{+}$ 466.9804, found 466.9805 .

2,2-双((2,5-二氧代吡咯烷-1-基) 氧基)乙酸乙酯 (3k): 白色固体，产率 50\%. m.p. 176 $177{ }^{\circ} \mathrm{C} ;{ }^{1} \mathrm{H} \mathrm{NMR}$ $\left(400 \mathrm{MHz}, \mathrm{CDCl}_{3}\right) \delta: 5.54(\mathrm{~s}, 1 \mathrm{H}), 4.35 \sim 4.29(\mathrm{~m}, 2 \mathrm{H})$, $2.70(\mathrm{~s}, 8 \mathrm{H}), 1.31(\mathrm{t}, J=7.2 \mathrm{~Hz}, 3 \mathrm{H}) ;{ }^{13} \mathrm{C}$ NMR (100 MHz, $\left.\mathrm{CDCl}_{3}\right) \delta: 170.1,161.8,104.3,63.2,25.4,13.9$; IR (KBr) $v: 3123,2980,1796,1756,1492,1373,1289,1181,995$, $879,698 \mathrm{~cm}^{-1}$; HRMS (ESI-TOF) calcd for $\mathrm{C}_{12} \mathrm{H}_{14} \mathrm{~N}_{2} \mathrm{NaO}_{8}$ $[\mathrm{M}+\mathrm{Na}]^{+}$337.0648, found 337.0646.

2,2-双((1,3-二氧代异吲哚啉-2-基)氧基)-2-(4-甲氧 苯基)乙酸乙酯(3I)：144 mg 白色固体，产率 56\%. m.p. $169 \sim 170{ }^{\circ} \mathrm{C} ;{ }^{1} \mathrm{H}$ NMR $\left(400 \mathrm{MHz}, \mathrm{CDCl}_{3}\right) \delta: 7.85 \sim 7.83$ $(\mathrm{m}, 2 \mathrm{H}), 7.82 \sim 7.72(\mathrm{~m}, 8 \mathrm{H}), 6.87(\mathrm{~d}, J=8.8 \mathrm{~Hz}, 2 \mathrm{H})$, 4.45 (d, $J=7.2 \mathrm{~Hz}, 2 \mathrm{H}), 3.79$ (s, 3H), 1.34 (t, $J=7.2 \mathrm{~Hz}$, $3 \mathrm{H}) ;{ }^{13} \mathrm{C}$ NMR (100 MHz, $\left.\mathrm{CDCl}_{3}\right) \delta: 164.8,162.9,161.3$, $134.5,129.8,128.9,123.6,123.4,113.4,110.2,63.2,55.3$, 13.9; IR (KBr) v: 3117, 2936, 1795, 1754, 1610, 1517, 1346, 1187, 1022, 982, 873, $698 \mathrm{~cm}^{-1}$; HRMS (ESI-TOF) calcd for $\mathrm{C}_{27} \mathrm{H}_{20} \mathrm{~N}_{2} \mathrm{NaO}_{9}[\mathrm{M}+\mathrm{Na}]^{+}$539.1066, found 539.1047.

2,2-双((1,3-二氧代异吲哚啉-2-基)氧基)-2-苯基乙 酸乙酯 $(3 \mathrm{~m})$ ： $112 \mathrm{mg}$ 白色固体，产率 46\%. m.p. 162 $163{ }^{\circ} \mathrm{C} ;{ }^{1} \mathrm{H}$ NMR (400 MHz, $\left.\mathrm{CDCl}_{3}\right) \delta: 7.95 \sim 7.91(\mathrm{~m}$, $2 \mathrm{H}), 7.79 \sim 7.72(\mathrm{~m}, 8 \mathrm{H}), 7.39 \sim 7.29(\mathrm{~m}, 3 \mathrm{H}), 4.43(\mathrm{~d}, J=$ $7.2 \mathrm{~Hz}, 2 \mathrm{H}), 1.32$ (t, $J=7.2 \mathrm{~Hz}, 3 \mathrm{H}) ;{ }^{13} \mathrm{C} \mathrm{NMR}(100 \mathrm{MHz}$, $\left.\mathrm{CDCl}_{3}\right) \delta: 164.5,162.8,134.5,131.6,130.9,128.9,128.0$, 128.0, 123.6, 110.2, 63.3, 13.9; IR (KBr) v: 3117, 2986, 1796, 1754, 1607, 1467, 1398, 1267, 1187, 1075, 873, 697 $\mathrm{cm}^{-1}$; HRMS (ESI-TOF) calcd for $\mathrm{C}_{26} \mathrm{H}_{18} \mathrm{~N}_{2} \mathrm{NaO}_{8}[\mathrm{M}+$ $\mathrm{Na}]^{+}$509.0961, found 509.0960.

2,2-双((1,3-二氧代异吲哚啉-2-基)氧基)-2-(4-氟苯 基)乙酸乙酯(3n): $144 \mathrm{mg}$ 白色固体, 产率 57\%. m.p. $180 \sim 181{ }^{\circ} \mathrm{C} ;{ }^{1} \mathrm{H}$ NMR (400 MHz, $\mathrm{CDCl}_{3}$ ) $\delta: 7.97 \sim 7.91$ $(\mathrm{m}, 2 \mathrm{H}), 7.83 \sim 7.74(\mathrm{~m}, 8 \mathrm{H}), 7.12 \sim 7.03(\mathrm{~m}, 2 \mathrm{H}), 4.46 \sim$ $4.40(\mathrm{~m}, 2 \mathrm{H}), 1.32(\mathrm{t}, J=7.2 \mathrm{~Hz}, 3 \mathrm{H}) ;{ }^{13} \mathrm{C} \mathrm{NMR}(100$ $\left.\mathrm{MHz}, \mathrm{CDCl}_{3}\right) \delta: 164.3,164.0\left(J_{\mathrm{CF}}=249.8 \mathrm{~Hz}\right), 162.9$, 134.6, $130.5\left(J_{\mathrm{CF}}=8.8 \mathrm{~Hz}\right), 128.9,127.7\left(J_{\mathrm{CF}}=3.3 \mathrm{~Hz}\right)$, 123.7, $115.2\left(J_{\mathrm{CF}}=21.8 \mathrm{~Hz}\right), 63.4,13.9$; IR (KBr) v: 3121 , 
1796, 1737, 1605, 1512, 1399, 1345, 1238, 1187, 1080, 991, 873, $697 \mathrm{~cm}^{-1}$; HRMS (ESI-TOF) calcd for $\mathrm{C}_{26} \mathrm{H}_{17} \mathrm{~F}-$ $\mathrm{N}_{2} \mathrm{NaO}_{8}[\mathrm{M}+\mathrm{Na}]^{+}$527.0867, found 527.0856.

2,2-双((1,3-二氧代异吲哚啉-2-基)氧基)-2-(4-氯苯 基)乙酸乙酯(3o): $127 \mathrm{mg}$ 白色固体, 产率 49\%. m.p. $178 \sim 179{ }^{\circ} \mathrm{C} ;{ }^{1} \mathrm{H}$ NMR $\left(400 \mathrm{MHz}, \mathrm{CDCl}_{3}\right) \delta: 7.94 \sim 7.88$ $(\mathrm{m}, 2 \mathrm{H}), 7.84 \sim 7.72(\mathrm{~m}, 8 \mathrm{H}), 7.41 \sim 7.33(\mathrm{~m}, 2 \mathrm{H}), 4.42 \sim$ $4.37(\mathrm{~m}, 2 \mathrm{H}), 1.29$ (t, $J=7.2 \mathrm{~Hz}, 3 \mathrm{H}) ;{ }^{13} \mathrm{C}$ NMR $(100$ $\left.\mathrm{MHz}, \mathrm{CDCl}_{3}\right) \delta: 164.1,162.9,137.0,134.7,130.5,129.6$, 128.8, 128.3, 123.7, 109.6, 63.5, 13.9; IR (KBr) v: 3099, 2980, 1796, 1755, 1596, 1495, 1405, 1289, 1188, 1097, $872,698 \mathrm{~cm}^{-1}$; HRMS (ESI-TOF) calcd for $\mathrm{C}_{26} \mathrm{H}_{17} \mathrm{ClN}_{2-}$ $\mathrm{NaO}_{8}[\mathrm{M}+\mathrm{Na}]^{+}$543.0571, found 543.0570.

2,2-双((1,3-二氧代异吲哚啉-2-基)氧基)-2-(4-溴苯 基)乙酸乙酯(3p): $149 \mathrm{mg}$ 白色固体, 产率 53\%. m.p. $180 \sim 181{ }^{\circ} \mathrm{C} ;{ }^{1} \mathrm{H}$ NMR $\left(400 \mathrm{MHz}, \mathrm{CDCl}_{3}\right) \delta: 7.90 \sim 7.68$ (m, $10 \mathrm{H}), 7.53$ (d, $J=8.8 \mathrm{~Hz}, 2 \mathrm{H}), 4.43 \sim 4.37(\mathrm{~m}, 2 \mathrm{H})$, 1.29 (t, $J=7.2 \mathrm{~Hz}, 3 \mathrm{H}) ;{ }^{13} \mathrm{C}$ NMR $\left(100 \mathrm{MHz}, \mathrm{CDCl}_{3}\right) \delta$ : 164.1, 162.9, 134.7, 131.4, 129.9, 128.9, 123.8, 63.5, 13.9; IR (KBr) v: 3371, 2859, 1777, 1467, 1400, 1293, 1188, $1077,989,873 \mathrm{~cm}^{-1}$; HRMS (ESI-TOF) calcd for $\mathrm{C}_{26} \mathrm{H}_{17^{-}}$ $\mathrm{BrN}_{2} \mathrm{NaO}_{8}[\mathrm{M}+\mathrm{Na}]^{+}$587.0066, found 587.0044.

2,2-双((2,5-二氧代-2,5-二氢-1 $H$-吡咯-1-基)氧基)-2苯基乙酸乙酯(3q): $105 \mathrm{mg}$ 白色固体, 产率 54\%. m.p. 200 201 ${ }^{\circ} \mathrm{C}$; ${ }^{1} \mathrm{H}$ NMR $\left(400 \mathrm{MHz}, \mathrm{CDCl}_{3}\right) \delta: 7.87$ (d, $J=$ $7.6 \mathrm{~Hz}, 2 \mathrm{H}), 7.57 \sim 7.36(\mathrm{~m}, 3 \mathrm{H}), 4.49 \sim 4.43(\mathrm{~m}, 2 \mathrm{H}), 2.63$ (s, 8H), 1.39 (t, $J=7.2 \mathrm{~Hz}, 3 \mathrm{H}) ;{ }^{13} \mathrm{C}$ NMR $(100 \mathrm{MHz}$, $\left.\mathrm{CDCl}_{3}\right) \delta: 170.3,164.5,131.5,131.2,128.5,128.0,63.3$, 25.4, 13.9; IR (KBr) v: 3117, 2986, 1732, 1696, 1426, 1238, 1089, 953, $856 \mathrm{~cm}^{-1}$; HRMS (ESI-TOF) calcd for $\mathrm{C}_{18} \mathrm{H}_{18} \mathrm{~N}_{2} \mathrm{NaO}_{8}[\mathrm{M}+\mathrm{Na}]^{+}$413.0961, found 413.0942.

2,2-双((2,5-二氧代吡咯烷-1-基)氧基)-2-(4-氟苯基) 乙酸乙酯(3t): $122 \mathrm{mg}$ 白色固体, 产率 60\%. m.p. 210 $211{ }^{\circ} \mathrm{C} ;{ }^{1} \mathrm{H}$ NMR $\left(400 \mathrm{MHz}, \mathrm{CDCl}_{3}\right) \delta: 7.92 \sim 7.88(\mathrm{~m}$, 2H), $7.11(\mathrm{t}, J=8.4 \mathrm{~Hz}, 2 \mathrm{H}), 4.46(\mathrm{~d}, J=7.2 \mathrm{~Hz}, 2 \mathrm{H}), 2.66$ (s, 8H), 1.39 (t, $J=7.2 \mathrm{~Hz}, 3 \mathrm{H}) ;{ }^{13} \mathrm{C}$ NMR $(100 \mathrm{MHz}$, $\left.\mathrm{CDCl}_{3}\right) \delta: 170.4,164.2,164.2\left(J_{\mathrm{CF}}=244 \mathrm{~Hz}\right), 130.9\left(J_{\mathrm{CF}}=\right.$ $8.9 \mathrm{~Hz}), 127.7,115.1\left(J_{\mathrm{CF}}=21.7 \mathrm{~Hz}\right), 107.7,63.4,25.5$, 13.9; IR (KBr) v: 3117, 2986, 1763, 1736, 1606, 1510, 1399, 1269, 1089, 996, 859, $647 \mathrm{~cm}^{-1}$; HRMS (ESI-TOF) calcd for $\mathrm{C}_{18} \mathrm{H}_{17} \mathrm{FN}_{2} \mathrm{NaO}_{8}[\mathrm{M}+\mathrm{Na}]^{+}$431.0867, found 431.0856 .

2,2-双((2,5-二氧代吡咯烷-1-基)氧基)-2-(4-氯苯基) 乙酸乙酯(3s): $119 \mathrm{mg}$ 白色固体, 产率 56\%. m.p. 212 $213{ }^{\circ} \mathrm{C} ;{ }^{1} \mathrm{H}$ NMR $\left(400 \mathrm{MHz}, \mathrm{CDCl}_{3}\right) \delta: 7.84$ (d, $J=8.8$
Hz, 2H), 7.39 (d, $J=8.8 \mathrm{~Hz}, 2 \mathrm{H}), 4.44$ (d, $J=7.2 \mathrm{~Hz}, 2 \mathrm{H})$, 2.67 (s, 8H), 1.37 (t, $J=7.2 \mathrm{~Hz}, 3 \mathrm{H}) ;{ }^{13} \mathrm{C}$ NMR $(100 \mathrm{MHz}$, $\left.\mathrm{CDCl}_{3}\right) \delta: 170.4,164.1,137.4,130.4,130.0,128.3,107.7$, 63.5, 25.5, 13.9; IR (KBr) v: 3117, 2994, 1749, 1717, 1496, 1426, 1295, 1213, 1091, 953, 835, $645 \mathrm{~cm}^{-1}$; HRMS (ESI-TOF) calcd for $\mathrm{C}_{18} \mathrm{H}_{17} \mathrm{ClN}_{2} \mathrm{NaO}_{8} \quad[\mathrm{M}+\mathrm{Na}]^{+}$ 447.0571, found 447.0552.

2,2-双((2,5-二氧代吡咯烷-1-基)氧基)-2-(4-溴苯基) 乙酸乙酯(3t): $122 \mathrm{mg}$ 白色固体, 产率 52\%. m.p. 204 $205{ }^{\circ} \mathrm{C} ;{ }^{1} \mathrm{H}$ NMR $\left(400 \mathrm{MHz}, \mathrm{CDCl}_{3}\right) \delta: 7.77$ (d, $J=8.8$ $\mathrm{Hz}, 2 \mathrm{H}), 7.55(\mathrm{~d}, J=8.8 \mathrm{~Hz}, 2 \mathrm{H}), 4.46 \sim 4.40(\mathrm{~m}, 2 \mathrm{H})$, 2.67 (s, 8H), 1.37 (t, $J=7.2 \mathrm{~Hz}, 3 \mathrm{H}) ;{ }^{13} \mathrm{C}$ NMR $(100 \mathrm{MHz}$, $\left.\mathrm{CDCl}_{3}\right) \delta: 170.4,164.0,131.2,130.9,130.2,125.8,107.7$, 63.5, 25.5, 13.9; IR (KBr) v: 3121, 1732, 1696, 1400, 1206, 1062, 999, 833, $668 \mathrm{~cm}^{-1}$; HRMS (ESI-TOF) calcd for $\mathrm{C}_{18} \mathrm{H}_{17} \mathrm{BrN}_{2} \mathrm{NaO}_{8}[\mathrm{M}+\mathrm{Na}]^{+}, 491.0066$, found 491.0045 .

辅助材料(Supporting Information) 化合物 $3 a \sim 3 t$ 的 ${ }^{1} \mathrm{H}$ NMR 和 ${ }^{13} \mathrm{C}$ NMR 图谱. 这些材料可以免费从本刊 网站(http://sioc-journal.cn/)上下载.

\section{References}

[1] (a) Doyle, M. P.; McKervey, M. A.; Ye, T. Modern Catalytic Methods for Organic Synthesis with Diazo Compounds, Wiley-Interscience, New York, 1998.

(b) Zhao, X.; Zhang, Y.; Wang, J. Chem. Commun. 2012, 48, 10162. (c) Zhu, S.-F.; Zhou, Q.-L. Nat. Sci. Rev. 2014, 1, 580.

(d) Gillingham, D.; Fei, N. Chem. Soc. Rev. 2013, 42, 4918.

(e) Ford, A.; Miel, H.; Ring, A.; Slattery, C. N.; Maguire, A. R.; Mckervey, M. A. Chem. Rev. 2015, 115, 9981.

(f) Zhang, Z.; Wang, J. Chem. Commun. 2009, 64, 5350.

(g) Doyle, M. P.; Duffy, R.; Ratnikov, M.; Zhou, L. Chem. Rev. 2010, 110, 704.

(h) Zhu, S.-F.; Zhou, Q.-L. Acc. Chem. Res. 2012, 45, 1365.

(i) Candeias, N.; Paterna, R.; Gois, P. M. P. Chem. Rev. 2016, 116, 2937.

(j) Zhang, T.-S.; Zhao, Q.; Hao, W.-J.; Tu, S.-J.; Jiang, B. Chem. Asian. J. 2019, 14, 1042.

(k) Zhang, D.; Hu, W. H. Chem. Rec. 2017, 17, 739.

(1) Qiu, D.; Qiu, M.; Ma, R.; Zhang, Y.; Wang, J. Acta Chim. Sinica 2016, 74, 472 (in Chinese).

(邱頔, 邱孟龙, 马戎, 张艳, 王剑波, 化学学报, 2016, 74, 472.)

[2] (a) Liu, L.; Zhang, J. Chin. J. Org. Chem. 2017, 37, 1117 (in Chinese).

(刘路, 张俊良, 有机化学, 2017, 37, 1117.)

(b) Wu, Y.-N.; Xu, T.; Fu, R.; Wang, N.-N.; Hao, W.-J.; Wang, S.-L.; Li, G.; Tu, S.-J.; Jiang, B. Chem. Commun. 2016, 52, 11943.

[3] (a) Clapham, B.; Spanka, C.; Janda, K. D. Org. Lett. 2001, 3, 2173. (b) Matsushita, H.; Lee, S.-H.; Yoshida, K.; Clapham, B.; Koch, G.; Zimmermann, J.; Janda, K. D. Org. Lett. 2004, 6, 4627.

[4] (a) Tan, F.; Liu, X.; Hao, X.; Tang, Y.; Lin, L.; Feng, X. ACS Catal. 2016, 6, 6930 .

(b) Zhang, Y.; Yao, Y.; He, L.; Liu, Y.; Shi, L. Adv. Synth. Catal. 2017, 359, 2754.

(c) Feng, J.; Yi, X.; Fu, Y.; Yu, Y.; Huang, F. Chin. J. Org. Chem. 2019, 39, 3013 (in Chinese). 
(封佳俊, 易享炎, 傅耀锋, 于杨, 黄菲, 有机化学, 2019，39, 3013.)

[5] (a) Maas, G. Chem. Soc. Rev. 2004, 33, 183.

(b) Sambasivanand, R.; Ball, Z. T. Angew. Chem., Int. Ed. 2012, 51, 8568 .

(c) Adly, F. G.; Gardiner, M. G.; Ghanem, A. Chem.-Eur. J. 2016, 22, 3447.

(d) Qin, C.; Boyarskikh, V.; Hansen, J. H.; Hardcastle, K. I.; Musaev, D. G.; Davies, H. M. L. J. Am. Chem. Soc. 2011, 133, 19198.

(e) Xu, H.; Li, Y.-P.; Cai, Y.; Wang, G.-P.; Zhu, S. F.; Zhou, Q.-L. J. Am. Chem. Soc. 2017, 139, 7697.

[6] (a) Padwa, A.; Weingarten, M. D. Chem. Rev. 1996, 96, 223.

(b) Yakura, T.; Ozono, A.; Matsui, K.; Yamashita, M.; Fujiwara, T. Synlett 2013, 24, 65.

(c) Roberts, E.; Sançon, J. P.; Sweeney, J. B. Org. Lett. 2005, 7, 2075.

[7] (a) Neupane, P.; Li, X.; Jung, J. H.; Lee, Y. R.; Kim, S. H. Tetrahedron 2012, 68, 2496.

(b) Dyer, J.; Jockusch, S.; Balsanek, V.; Sames, D.; Turro, N. $J$. Org. Chem. 2005, 70, 2143 .

(c) Dussault, P. H.; Xu, C. Tetrahedron Lett. 2004, 45, 7455.

[8] (a) Wang, N.-N.; Huang, L.-R.; Hao, W.-J.; Zhang, T.-S.; Li, G.; Tu, S.-J.; Jiang, B. Org. Lett. 2016, 18, 1298.

(b) Hao, W.-J.; Gao, Q.; Jiang, B.; Liu, F.; Wang, S.-L.; Tu, S.-J.; Li, G. J. Org. Chem. 2016, 81, 11276.

(c) Wang, N.-N.; Hao, W.-J.; Zhang, T.-S.; Li, G.; Wu, Y.-N.; Tu, S.-J.; Jiang, B. Chem. Commun. 2016, 52, 5144.

(d) Zhang, T.-S.; Hao, W.-J.; Wang, N.-N.; Li, G.; Jiang, D.-F.; Tu, S.-J.; Jiang, B. Org. Lett. 2016, 18, 3078.

(e) Zhang, T.-S.; Zhang, H.; Fu, R.; Wang, J.; Hao, W.-J.; Tu, S.-J.; Jiang, B. Chem. Commun. 2019, 55, 13231.

[9] (a) Jiang, B.; Li, J.; Pan, Y.-Y.; Hao, W.-J.; Li, G.; Tu, S.-J. Chin. J. Chem. 2017, 35, 323.

(b) Qiu, J.-K.; Jiang, B.; Zhu, Y.-L.; Hao, W.-J.; Wang, D.-C.; Sun, J.; Wei, P.; Tu S.-J.; Li, G. J. Am. Chem. Soc. 2015, 137, 8928.

(c) Huang, M.-H.; Hao, W.-J.; Li, G.; Tu, S.-J.; Jiang, B. Chem. Commun. 2018, 54, 10791.

(d) Huang, M.-H.; Hao, W.-J.; Jiang, B. Chem. Asian J. 2018, 13, 2958

(e) Peng, H. H.; Yuan, Z. L.; Chen, P. H.; Liu, G. S. Chin. J. Chem. 2017, 35, 876 .

(f) Song, H.; Liu, X. Y.; Qin, Y. Acta Chim. Sinica 2017, 75, 1137 (in Chinese).

(宋影, 刘小宇, 秦勇, 化学学报, 2017, 75, 1137.) (g) Wei, W.; Wang, L.; Bao, P.; Shao, Y.; Yue, H.; Yang, D.; Yang, X.; Zhao, X.; Wang H. Org. Lett. 2018, 20, 7125.

(i) Wang, L.; Bao, P.; Liu, W.; Liu, S.; Hu, C.; Yue, H.; Yang, D.; Wei, W. Chin. J. Org. Chem. 2018, 38, 3189 (in Chinese). (王雷雷, 鲍鹏丽, 刘维伟, 刘思殁, 胡昌松, 岳会兰, 杨道山, 魏伟, 有机化学, 2018, 38, 3189.)

[10] (a) Lu, F.-D.; Jiang, X.; Lu, L.-Q.; Xiao, W.-J. Acta Chim. Sinica 2019, 77, 803 (in Chinese).

(路福东，姜烜，陆良秋，肖文精，化学学报, 2019, 77, 803.)

(b) Hai, M.; Guo, L.-N.; Wang, L.; Duan, X.-H. Acta Chim. Sinica 2019, 77, 895 (in Chinese)

(海曼, 郭丽娜, 王乐, 段新华, 化学学报, 2019, 77, 895.)

(c) Yang, J.; Fu, X.; Lu, Z.; Zhu, G. Acta Chim. Sinica 2019, 77, 901 (in Chinese).

(杨俊航, 傅晓波, 卢增辉, 朱钢国, 化学学报, 2019, 77, 901.)

(d) Lin, F.; Liang, Y.; Li, X.; Song, S.; Jiao, N. Acta Chim. Sinica 2019, 77, 906 (in Chinese)

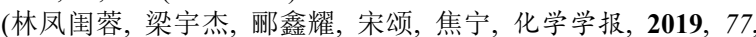
906.)

(e) Tang, N.; Shao, X.; Wang, M.; Wu, X.; Zhu, C. Acta Chim. Sinica 2019, 77, 922 (in Chinese).

(汤娜娜，郡金金，王明扬，吴新釒金，朱晨，化学学报，2019，77, 922.)

(f) Zhao, Q.; Tu, S.-J.; Jiang B. Acta Chim. Sinica 2019, 77, 927 (in Chinese).

(赵琦, 屠树江, 姜波, 化学学报, 2019, 77, 927.)

(g) Fu, R.; Li, M.-F.; Zhou, P.; Hao, W.-J.; Tu, S.-J.; Jiang, B. Adv. Synth. Catal. 2019, 361, 2280.

[11] (a) Lu, H.; Dzik, W. I.; Xu, X.; Wojtas, L.; de Bruin, B.; Zhang, X. P. J. Am. Chem. Soc. 2011, 133, 8518

(b) Zheng, J.; Qi, J.; Cui, S. J. Org. Chem. 2015, 80, 9224

(c) Jiang, J.; Liu, J.; Yang, L.; Shao, Y.; Cheng, J.; Bao, X.; Wan, X. Chem. Commun. 2015, 51, 14728.

[12] Zhang, J.; Jiang, J.; Xu, D.; Luo, Q.; Wang, H.; Chen, J.; Li, H.; Wang, Y.; Wan, X. Angew. Chem., Int. Ed. 2015, 54, 1231

[13] Wang, N.-N.; Hao, W.-J.; Zhang, T.-S.; Li, G.; Wu, Y.-N.; Tu, S.-J.; Jiang, B. Chem. Commun. 2016, 52, 5144.

[14] (a) Lu, Y.; Han, Y.; Sun, Y.; Abdukader, A.; Wang, D.; Liu, C. Chin. J. Org. Chem. 2020, 40, 447 (in Chinese).

(陆雨函，韩颖芝，孙亚栋，阿布力米提・阿布都卡德尔，王多志， 刘晨江, 有机化学, 2020, 40, 447.)

(b) Chen, Q.; Yang, Y.; Wang, X.; Zhang, Q.; Li, D. Chin. J. Org. Chem. 2020, 40, 454 (in Chinese).

(陈倩雯, 杨耀成, 王霞, 张谦, 李栋, 有机化学, 2020, 40, 454.)

(Li, L.; Fan, Y.) 